\title{
Arginase levels and their association with Th17-related cytokines, soluble adhesion molecules (sICAM-1 and SVCAM-1) and hemolysis markers among steady-state sickle cell anemia patients
}

\author{
Wendell Vilas-Boas • Bruno A. V. Cerqueira • \\ Angela M. D. Zanette • Mitermayer G. Reis • \\ Manoel Barral-Netto • Marilda S. Goncalves \\ Received: 13 January 2010 /Accepted: 23 March 2010 / Published online: 20 April 2010 \\ (C) The Author(s) 2010. This article is published with open access at Springerlink.com
}

\begin{abstract}
Sickle cell anemia (SCA) is characterized by a marked endothelial dysfunction, owing to many factors. Arginine metabolism can be related to the inflammatory chronic state presented by patients, playing a key role in their clinical outcome and vascular endothelium. We investigated the serum arginase levels in 50 SCA patients (22 men and 28 women, mean age of $17 \pm 10.5$ years) and 28 healthy controls. Serum arginase levels were associated with biochemical hemolysis markers and cytokines involved in Th17 response, as well as levels of soluble intercellular adhesion molecule-1 (sICAM-1) and vascular cell adhesion molecule-1 (sVCAM-1). Arginase concentrations were higher in SCA patients, compared with controls $(p=0.005)$, and were significantly and positively associated with total bilirubin $(p=0.004)$, indirect bilirubin $(p=0.04)$, and aspartate aminotransferase (AST; $p=0.039$ ) in the SCA patient group. Moreover, arginase was significantly and positively associated with transforming
\end{abstract}

WVB and BAVC contributed equally to this manuscript.

W. Vilas-Boas · B. A. V. Cerqueira · M. G. Reis ·

M. Barral-Netto $\cdot$ M. S. Goncalves $(\bowtie)$

Centro de Pesquisas Gonçalo Moniz/FIOCRUZ,

Rua Waldemar Falcão 121. Brotas,

Salvador, Bahia CEP. 40.295-001, Brazil

e-mail: mari@bahia.fiocruz.br

M. S. Goncalves

Departamento de Analises Clínicas e Toxicologicas/Faculdade de Farmácia, Universidade Federal da Bahia,

Salvador, Brazil

A. M. D. Zanette

Fundacao de Hematologia e Hemoterapia da Bahia-Hemoba,

Salvador, Brazil growth factor-beta (TGF-beta; $p=0.008$ ) among SCA patients. sICAM-1 was significantly and positively associated to reticulocytes $(p=0.014)$ and AST $(p=0.04)$. sVCAM-1 was likewise associated with lactate dehydrogenase $(p=0.03)$. These data suggest a new insight into arginase metabolism, as we show here a shift in arginine catabolism, where TGF-beta may induces the arginase pathway instead of the nitric oxide pathway and a possible involvement of the vascular activation and the serum arginase in chronic hemolysis among SCA patients. Additional studies should be carried out in order to investigate the mechanisms by which TGF-beta participates in the metabolism of arginase in SCA patients.

Keywords Sickle cell anemia · Arginine - Arginase · Th17 cells $\cdot$ Soluble adhesion molecules $\cdot$ TGF- $\beta$

\section{Introduction}

Sickle cell anemia (SCA) has been characterized by a chronic inflammatory state, with the presence of elevated leukocyte counts, abnormal activation of granulocytes, monocytes and endothelial cells, and an increased level of multiple inflammatory mediators. Cytokine expression may play a role in the clinical outcome of SCA patients. Its effects on vascular endothelium and adhesion molecule expression have been considered relevant [1-3].

Normal arginine metabolism is impaired in SCA, contributing to endothelial dysfunction and pulmonary hypertension [4]. Arginase catalyzes the hydrolysis of Larginine into L-ornithine and urea. It is widely distributed in living organisms, utilizing arginine as its substrate [5]. In 
patients with SCA, and likely other hemolytic conditions, intravascular hemolysis contributes to endothelial dysfunction, which has been characterized by a reduction in nitric oxide (NO) bioavailability and NO resistance [6]. Given the important role of NO depletion in endothelial dysfunction, it is not surprising that NO dysregulation is a common finding among the varied mechanisms involved in sickle vasculopathy [7]. Arginase can be induced in many cell types by a variety of cytokines and inflammatory stimuli $[8,9]$.

Interleukin 17 (IL-17) is an essential proinflammatory Tcell-derived cytokine with several biological actions. It has been found to play a pivotal role in microbial host defense by interconnecting lymphoid and myeloid host defenses [10]. While previously considered to be dependent on interleukin-23 (IL-23) for differentiation from naive T cells into Th17 cells, recent work has indicated that initial differentiation is dependent on transforming growth factorbeta (TGF-beta), with subsequent expansion of the Th17 lineage dependent on IL-23 [11]. The role of the Th17 response in vascular alterations has not been established. Taleb et al. [12] showed that in vivo administration of IL-17 reduces endothelial vascular cell adhesion molecule-1 (VCAM-1) expression and vascular T-cell infiltration, and significantly limits the development of atherosclerotic lesions. However, Eid et al. [13] showed that IL-17 is produced concomitantly with interferon gamma (IFNgamma) by coronary-artery-infiltrating $\mathrm{T}$ cells, which act synergistically to induce proinflammatory responses in vascular smooth muscle cells.

In macrophages, arginase gene expression is tightly regulated by many factors, including exogenous stimuli like the Th2 cytokines [14]. TGF-beta reduces the inducible NO synthase activity of rat macrophage cultures, not only down-regulating the inducible nitric oxide synthase pathway, but also up-regulating the arginase pathway [15].

In this study we tested the hypothesis that serum arginase concentration is influenced by some factors such as hemolysis, and Th17 responses and is related to endothelial dysfunction. We have tested that by evaluating hemolysis markers as bilirubin, lactate dehydrogenase (LDH) and aminotransferase, as well as cytokines involved in the Th17 response (IL-23, IL-17, and TGF-beta) and soluble adhesion molecules levels [soluble intercellular adhesion molecule-1 (sICAM-1) and vascular cell adhesion molecule-1 (sVCAM-1)].

\section{Patients and methods}

\section{Subjects}

We studied 50 patients ( 22 men and 28 women, mean age of $17 \pm 10.5$ years) from Northeast Brazil who were diagnosed with SCA (HBSS) and were located at the Foundation of Hematology and Hemotheraphy (HEMOBA) in Bahia, Brazil. All patients were at a steady state and were transfusion free. Patients showed no other systemic diseases that could have potentially altered their inflammatory profile functions. Blood samples were obtained during regular clinical visits. Twenty-eight healthy Brazilian subjects with normal hemoglobin profiles were included as a control group. The study was approved by the Oswaldo Cruz Foundation's Human Research Board and is in accordance with the Declaration of Helsinki of 1975, as revised in 2000. All subjects signed an informed consent form.

Biochemical analyses and serum arginase level measurements

Serum concentrations of bilirubin, LDH, aspartate aminotransferase (AST), and alanine aminotransferase (ALT) were determined using commercially available biochemical kits (LABTEST, Minas Gerais, Brazil). Arginase serum levels in SCA patients and control groups were measured using a Human Arginase I ELISA Kit (Cell science, Canton, MA, USA) according to the manufacturer's recommendations.

Cytokine and soluble adhesion molecule measurements

IL-23, IL-17, and TGF-beta were measured using Cytokine ELISA OptEIA kits (BD Pharmingen, San Diego, CA, USA) and soluble adhesion molecules were characterized using ELISA Kits (R\&D Systems, Minneapolis, MN, USA), according to the manufacturer's recommendations.

\section{Statistical analysis}

Baseline characteristics were summarized as means and proportions of selected variables. The distribution of quantitative variables was determined using the Kolmogorov-Smirnov test. Mean values of quantitative variables between groups were compared using the unpaired $t$ test for normal data distribution and Mann-Whitney for nonnormal data. The Kruskal-Wallis test or ANOVA with Bonferoni were used to compare means among two or more groups, as measured by interval variables. Bivariate correlation analyses were carried out to determine correlations between pairs of variables using Pearson's and Spearman's rank correlations $(r)$. Linear regression analyses were also performed. All tests were considered significant if $p$ values were less than 0.05 . Data analyses were performed using Prism 5.01 (Graphpad Software, San Diego, CA, USA), EPIinfo 6.04 (CDC, Atlanta, Georgia, USA), and STATA SE 10 software (StataCorp, Texas, USA). 


\section{Results}

Arginase levels were higher in SCA patient sera compared to controls

Serum arginase levels were quantified to determine the amount of arginase released into the blood. Arginase serum levels in SCA patients showed a twofold increase in enzyme levels, compared with control patients (median 21.1 and $14.7 \mathrm{ng} / \mathrm{mL}$, respectively). This was found to be statistically significant ( $p=0.005$; Fig. 1 ). Table 1 shows the hematologic and hemolysis markers values of the SCA patients group.

Correlation of arginase levels and hemolytic biochemical markers

The association between serum arginase concentrations and clinical hemolysis laboratory markers was determined to confirm the mechanisms that increase enzyme levels in SCA patients. Serum arginase levels were positively and significantly associated with total bilirubin $(p=0.004, r=$ 0.397 ), indirect bilirubin ( $p=0.040, r=0.291)$, and AST ( $p=$ $0.024, r=0.320)$. Correlation analysis of AST showed a positive association with $\mathrm{LDH}(p<0.001, r=0.59)$ and total bilirubin $(p<0.001, r=0.49)$.

Arginase was positively and significantly associated with a high expression of TGF-beta among SCA patients

In this work, we investigated cytokines involved in Th17 response and arginase expression regulation (e.g., IL-23,

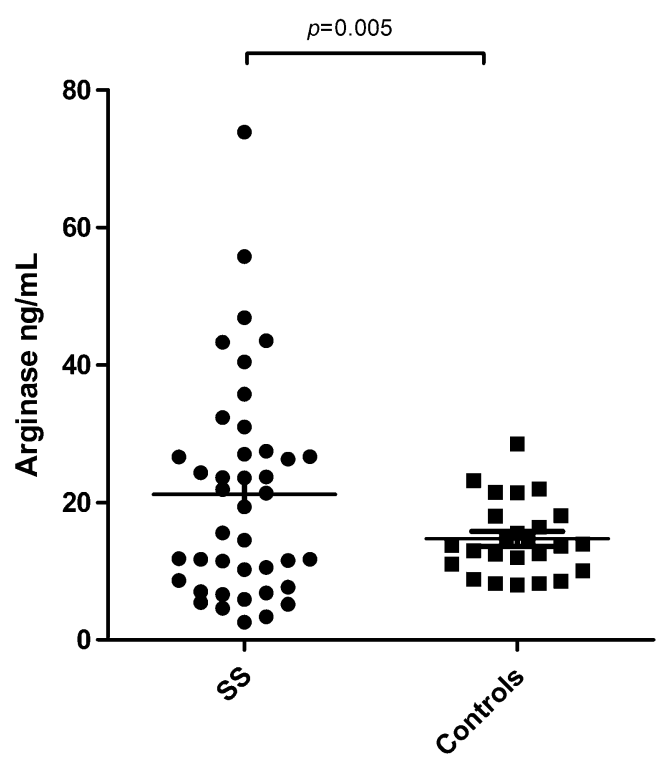

Fig. 1 Arginase serum levels between sickle cell anemia patients and control groups
Table 1 Steady-state hematologic and hemolysis markers values of the SCA patients group

\begin{tabular}{lcc}
\hline & Median & SD \\
\hline Hematology values & & \\
RBC $\left(\times 10^{6} / \mu \mathrm{L}\right)$ & 2.69 & 0.67 \\
Hemoglobin $(\mathrm{g} / \mathrm{dL})$ & 8.07 & 1.60 \\
Hematocrit $(\%)$ & 24.42 & 5.26 \\
Platelets $\left(\times 10^{3} / \mu \mathrm{L}\right)$ & 368 & 178 \\
Reticulocytes count $(\%)$ & 6.9 & 3.1 \\
Hemolysis markers & & \\
Total bilirubin $(\mathrm{mg} / \mathrm{dL})$ & 2.35 & 1.74 \\
Direct bilirubin $(\mathrm{mg} / \mathrm{dL})$ & 0.72 & 0.61 \\
Indirect bilirubin $(\mathrm{mg} / \mathrm{dL})$ & 1.64 & 1.35 \\
AST $(\mathrm{U} / \mathrm{l})$ & 44.55 & 29.96 \\
ALT $(\mathrm{U} / \mathrm{l})$ & 11.25 & 9.63 \\
\hline
\end{tabular}

$S D$ standard deviation, $A S T$ aspartate aminotransferase, $A L T$ alanine aminotransferase

IL-17, and TGF-beta), associating these with free arginase in SCA individuals. There was a positive and significant association between arginase and TGF-beta $(p=0.008, r=$ 0.588 ), as shown in Table 2. The linear regression of arginase levels was also positively and significantly associated with AST $(p<0.0001, r=0.522)$, total bilirubin $(p=0.034, r=0.301)$, and indirect bilirubin $(p=0.039, r=$ 0.293; Fig. 2).

Rises in hemolytic rate is correlated to SVCAM-1 and sICAM-1 expression

We also studied vascular and leukocyte activation using sVCAM-1 and sICAM-1 serum level measurements. We found a positive and significant correlation among SICAM-

Table 2 Correlation between arginase serum levels, TH17-related cytokine, and soluble adhesion molecules levels in SCA patients

\begin{tabular}{lrr}
\hline \multicolumn{2}{c}{ ARGINASE } \\
\hline Cytokine & $p$ value & $r$ \\
IL-23 & 0.459 & 0.181 \\
IL-4 & 0.684 & -0.100 \\
IL-17 & 0.110 & 0.378 \\
TGF-beta & $0.008^{\mathrm{a}}$ & 0.588 \\
Soluble adhesion molecules & & \\
sICAM-1 & 0.192 & -0.313 \\
sVCAM-1 & 0.303 & 0.249 \\
\hline
\end{tabular}

${ }^{\mathrm{a}}$ Spearman or Pearson correlation coefficient $(r)$ and $p$ values

$s I C A M-1$ soluble intracellular adhesion molecule-1, $s$ VCAM-1 soluble vascular cell adhesion molecule-1, TGF-beta transforming growth factor-beta 

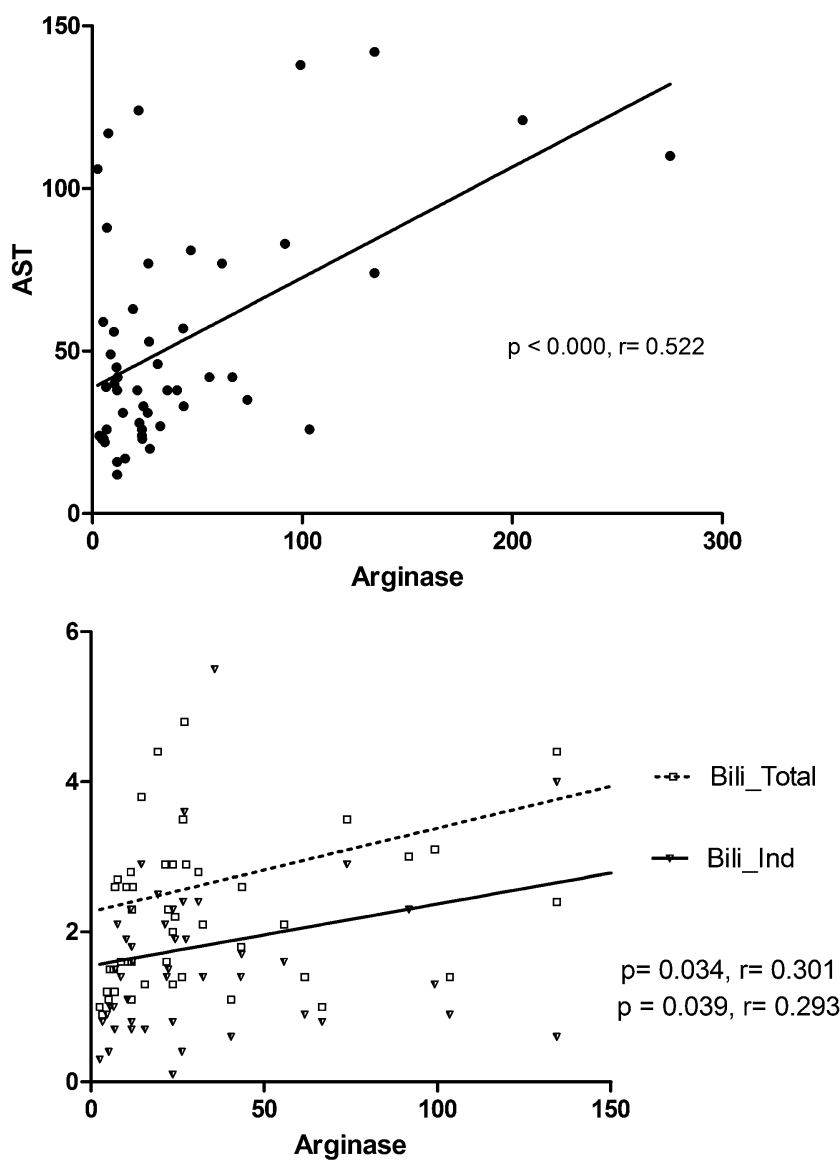

Fig. 2 Linear regression of arginase serum $(\mathrm{ng} / \mathrm{mL})$ and hemolysis markers in sickle cell anemia patients

1 and reticulocyte count $(p=0.014, r=0.270)$, as well as with $\operatorname{AST}(p=0.04, r=0.227)$, which are both classical hemolysis markers. Moreover, soluble VCAM-1 levels were associated with an increase in LDH levels $(p=0.041$, $r=0.28)$. No association was found between arginase and sVCAM-1 or sICAM-1 (Table 2).

\section{Discussion}

Results presented here demonstrate that free arginase levels in the sera of SCA patients are markedly increased when compared with healthy individuals. This is in agreement, in part, with a previous report that estimated the rise in arginase activity for SCA patients [4], while we have investigated the enzyme concentration and not its activity. A high concentration of free arginase in plasma is supposed to deplete the circulating L-arginine. It is known that adults with sickle cell disease are arginine deficient when in a steady state, also, plasma arginine concentrations significantly decrease in both adults and children during vasoocclusive crisis and acute chest syndrome, and this effect is associated with low NO metabolite levels [16]. Clinical trials with L-arginine supplementation appear to correct the NO deficiency and ameliorate pulmonary hypertension. In addition, red-cell adherence to the pulmonary endothelium appears to decrease with increasing NO [17, 18].

During hemolysis, free hemoglobin is decompartmentalized from the erythrocyte and is released into plasma, where it rapidly reacts with and destroys NO. This contributes to an abnormally high $\mathrm{NO}$ consumption and the formation of reactive oxygen species, ultimately inhibiting vasodilation. NO destruction by free hemoglobin can also contribute to impairment of vascular endothelial function via transcriptional activation of adhesion molecules, including VCAM-1 and E-selectin, as well as potent vasoconstrictors such as endothelin-1 [19]. Results presented here strongly suggest that the major source of free serum arginase released into plasma was the erythrocyte, as we found a positive correlation between hemolytic biochemical markers and arginase. Thus, a worsening in the clinical outcome can be observed by the simultaneous release of erythrocyte arginase during hemolysis, which will also limit the availability of arginine to NOS, contributing to a NO deficiency $[4,19]$. In fact, this observation was supported by results from sVCAM-1 and SICAM-1, which were positively associated with hemolysis markers, such as AST, LDH, and reticulocyte count.

Characterizing NO metabolism is an important goal in SCA studies, as NO regulates blood vessel tone, endothelial adhesion, leukocytes, and platelet activity. These are important factors in ischemia-reperfusion injury and sickle-cell-induced ischemia. In sickle cell anemia more adhesion molecules are produced owing to a decreased availability of NO [20]. As part of the biochemical program that facilitates blood flow and maintains vascular homeostasis, NO normally suppresses the expression of VCAM-1, ICAM-1, and E-selectin. In fact, the impairment of NO bioavailability, which is associated with endothelial dysfunction, results in the pathological activation of endothelial cells to express adhesion molecules [21], contributing to new vaso-occlusive events. Morris et al. [4] showed an association between sVCAM-1 and arginase levels. NO acts as an anti-inflammatory mediator, affecting endothelial cell function by preventing leukocyte adhesion to the endothelium through the inhibition of adhesion molecule expression [22]. However, no correlation was found between such molecules in our results. This might be due to the clinical state of patients, who were in a steady state of the disease, at the moment of blood collection.

We also investigated the association of cytokines related to Th17 response and the extent of arginase release into the plasma. There is not much information concerning this topic in literature, and it has not been presented in the 
context of SCA. Among the studied cytokines, there is no association between IL-23 and IL-17 concentrations with arginase levels. However, SCA individuals with increased levels of TGF-beta had the highest levels of serum arginase. This is an intriguing result, considering that the NO metabolic pathway in murine macrophages is a key defense element for various infectious diseases. Also, its role in diverse settings of immunopathology has been firmly established [23]. Arginase activity in macrophages is exclusively up-regulated in association with cytokines involved in the Th2 immune response, including the cytokines IL-4 and IL-10 [9, 24], as well as IL-13 and TGF-beta [25]. TGF-beta has effects on cell proliferation and apoptosis, as well as on the response to tissue injury, infection, bone homeostasis, endothelial growth, pulmonary fibrosis, inflammation, immune regulation, and extracellular matrix synthesis [26]. Our work reinforces the model that the arginase pathway is regulated by TGF-beta. This is true not only for its immune response role, as previously described, but also in other types of tissue that are involved in arginase metabolism. Naive Tcell differentiation into Th17 cells is driven initially by TGF-beta [11]. The lack of association among arginase and Th17-related cytokines may be due to a secondary role that these cytokines can perform in arginine metabolism, mainly when patients are in a steady state of the disease.

The clinical outcomes of SCA patients are a consequence of several events and can also be influenced by genetic diversity. With this respect, the reciprocal regulation of the NOS/arginase pathway that results in a metabolic shift in arginine utilization, a key endogenous substrate of both enzymes, may represent an important target to modulate clinical symptoms and a further therapeutic approach for SCA patients.

Acknowledgments This work was supported by grants from the Brazilian National Council of Research (CNPq; 3065427/2007-5 and 484457/2007-1; M.S.G.), the Foundation of Research of Bahia (FAPESB; 1431040053063 and 9073/2007; M.S.G.). The sponsors of this study are public or nonprofit organizations that support science in general. They had no role in gathering, analyzing, or interpreting the data.

Open Access This article is distributed under the terms of the Creative Commons Attribution Noncommercial License which permits any noncommercial use, distribution, and reproduction in any medium, provided the original author(s) and source are credited.

\section{References}

1. Hebbel RP, Osarogiagbon R, Kaul D (2004) The endothelial biology of sickle cell disease: inflammation and a chronic vasculopathy. Microcirculation 11:129-151
2. Okpala I (2004) The intriguing contribution of white blood cells to sickle cell disease - a red cell disorder. Blood Rev 18:65-73. doi:10.1016/S0268-960X(03)00037-7

3. Telen MJ (2007) Role of adhesion molecules and vascular endothelium in the pathogenesis of sickle cell disease. Hematology Am Soc of Hematol Educ Program 84-90

4. Morris CR, Kato GJ, Poljakovic M et al (2005) Dysregulated arginine metabolism, hemolysis-associated pulmonary hypertension and mortality in sickle cell disease. JAMA 294:81-90. doi:10.1001/jama.294.1.81

5. Iyamu EW, Cecil R, Parkin L, Woods G, Ohene-Frempong K, Asakura T (2005) Modulation of erythrocyte arginase activity in sickle cell disease patients during hydroxyurea therapy. Br J Haematol 131:389-394. doi:10.1111/j.1365-2141.2005.05772

6. Gladwin MT, Kato GJ (2005) Cardiopulmonary complications of sickle cell disease: role of nitric oxide and hemolytic anemia. Hematology Am Soc Hematol Educ Program 51-57 doi:10.1182/ asheducation-2005.1.51

7. Wood KC, Hsu LL, Gladwin MT (2008) Sickle cell disease vasculopathy: a state of nitric oxide resistance. Free Radic Biol Med 44:1506-1528. doi:10.1016j.freeradbiomed.2008.01.0085

8. Morris SM Jr (2002) Regulation of enzymes of the urea cycle and arginine metabolism. Annu Rev Nutr 22:87-105. doi:10.1146/ annurev.nut.22.110801.140547

9. Corraliza IM, Soler G, Eichmann K, Modolell M (1995) Arginase induction by suppressors of nitric oxide synthesis (IL-4, IL-10 and PGE2) in murine bone marrow-derived macrophages. Biochem Biophys Res Commun 206:667-673

10. Schwarzenberger P, Kolls JK (2002) Interleukin 17: an example for gene therapy as a tool to study cytokine mediated regulation of hematopoiesis. J Cell Biochem 38(Suppl):88-95

11. Stockinger B, Veldhoen M (2007) Differentiation and function of Th17 cells. Curr Opin Immunol 19:281-286. doi:10.10161j. coi.2007.04.005

12. Taleb S, Romain M, Ramkhelawon B, Uyttenhove C, Pasterkamp G, Herbin O et al (2009) Loss of SOCS3 expression in T cells reveals a regulatory role for interleukin-17 in atherosclerosis. $\mathrm{J}$ Exp Med 206:2067-2077. doi:10.1084/jem.20090545

13. Eid RE, Rao DA, Zhou J, Lo SF, Ranjbaran H, Gallo A et al (2009) Interleukin-17 and interferon-gamma are produced concomitantly by human coronary artery-infiltrating $\mathrm{T}$ cells and act synergistically on vascular smooth muscle cells. Circulation 119:1424-1432. doi:101161/circulationaha.108.827618

14. Pauleau AL, Rutschman R, Lang R, Pernis A, Watowich SS et al (2004) Enhancer-mediated control of macrophage-specific arginase I expression. J Immunol 172:7565-7573

15. Mills CD, Kincaid K, Alt JM, Heilman MJ, Hill AM (2000) M-1/ M-2 macrophages and the Th1/Th2 paradigm. J Immunol 164:6166-6173

16. Morris CR, Kuypers FA, Larkin S, Vichinsky E, Styles L (2000) Patterns of arginine and nitric oxide in sickle cell disease patients with vaso-occlusive crisis and acute chest syndrome. J Pediatr Hematol Oncol 22:515-520

17. Morris CR, Morris SM Jr, Hagar W, Van Warmerdam J, Claster S, Kepka-Lenhart D, Machado L, Kuypers FA, Vichinsky EP (2003) Arginine therapy: a new treatment for pulmonary hypertension in sickle cell disease? Am J Respir Crit Care Med 168:63-69. doi:10.1164/rccm.200208-967OC

18. Morris CR, Kuypers FA, Larkin S et al (2000) Arginine therapy: a novel strategy to induce nitric oxide production in sickle cell disease. Br J Haematol 111:498-500. doi:10.1111/j.1365-2141.2000.12403

19. Rother RP, Bell L, Hillmen P, Gladwin MT (2005) The clinical sequelae of intravascular hemolysis and extracellular plasma hemoglobin: a novel mechanism of human disease. JAMA 293:1653-1662

20. Vichinsky E (2002) New therapies in sickle cell disease. Lancet 360:629-631 
21. Lee SK, Kim JH, Yang WS, Kim SB, Park SK, Park JS (2002) Exogenous nitric oxide inhibits VCAM-1 expression in human peritoneal mesothelial cells. Role of cyclic GMP and NF-kappaB. Nephron 90:447-454. doi:10.1159/000054733

22. Nabah YN, Mateo T, Cerda-Nicolas M, Alvarez A, Martinez M, Issekutz AC, Sanz MJ (2005) L-NAME induces direct arteriolar leukocyte adhesion, which is mainly mediated by angiotensin-II. Microcirc 12:443-453. doi:10.1080/10739680590960962

23. MacMicking J, Xie QW, Nathan C (1997) Nitric oxide and macrophage function. Annu Rev Immunol 15:323-350. doi:10.1146/annurev.immunol.15.1.323
24. Modolell M, Corraliza IM, Link F, Soler G, Eichmann K (1995) Reciprocal regulation of nitric oxide synthase/arginase balance in mouse bone marrow-derived macrophages by Th1 and Th2 cytokines. Eur J Immunol 25:1101-1104

25. Munder M, Eichmann K, Modolell M (1998) Alternative metabolic states in murine macrophages reflected by the nitric oxide synthase/ arginase balance: competitive regulation by $\mathrm{CD} 4+\mathrm{T}$ cells correlates with Th1/Th2 phenotype. J Immunol 160:5347-5354

26. Ziyadeh FN (2004) Mediators of diabetic renal disease: the case for TGF-Beta as the major mediator. J Am Soc Nephrol 15(Suppl 1): S55-S57. doi:10.1097/01.asn.0000093460.24823.5B 